\title{
Endovascular Ischemic Stroke Models in Nonhuman Primates
}

\author{
Di Wu ${ }^{1,2,3} \cdot$ Ankush Chandra ${ }^{4,5}$. Jian Chen ${ }^{1,6} \cdot$ Yuchuan Ding ${ }^{1,4} \cdot$ Xunming Ji $\mathbf{1}^{1,2,3}$
}

Published online: 8 November 2017

(C) The American Society for Experimental NeuroTherapeutics, Inc. 2017
Key Words Ischemic stroke · animal model · thrombolysis · prognosis $\cdot$ nonhuman primate

\section{Introduction}

Cerebral stroke is one of the leading causes of death and disability, accounting for almost $9.6 \%$ of all deaths worldwide [1]. In the USA, someone has a stroke every $40 \mathrm{~s}$ and somebody dies from this disease every $4 \mathrm{~min}$, demonstrating the omnipresence, frequency, and lethality of this disease [2]. Stroke can broadly be divided in to ischemic and hemorrhagic, with diverse clinical and radiographic presentations. Ischemic stroke is a highly complex and heterogeneous disorder, which causes neurological damage and dysfunction due to lack of oxygen delivery to the brain. The final infarct size and the neurological outcome depend on a multitude of factors, including duration and severity of ischemia; the existence of collateral vascular systems; and localization of the infarct, such as the proximal end of intracranial artery (ICA), the main trunk of middle cerebral artery (MCA), and the branch of MCA (M2) [3]. In spite of the pervasiveness of this disease, only tissue plasminogen activator (t-PA) has been approved by the Food and Drug Administration for the acute treatment of ischemic stroke [4]. Therefore, translation from bench research to bedside treatment of patients with ischemic stroke is a necessity for newer and more effective therapies $[5,6]$. Proposed recommendations to improve the translation of acute stroke therapies have included evaluation of agents in multiple animal models of stroke, especially in nonhuman primates (NHPs) [7].

NHPs are unique owing to their phylogenetic proximity to humans, and similar physiological functions and life course (just on a shorter time scale) to humans, which provide an advantage in translational and preclinical biological studies 
[8]. Similarities in life longevity, age-related disorders, and cognitive abilities between NHPs and humans are inherent advantages for these animal models in translational medical studies $[9,10]$. Moreover, similar brain size, anatomy, and compositions have made the use of radiology a strong tool in research with NHPs $[9,10]$. In fact, owing to their similarity to humans, many human diseases, including coronary sclerosis, emphysema, degenerative joint diseases, cancer, and diabetes, have been found and reported in the middle-aged and old NHPs $[11,12]$. For these reasons, NHPs have been described to be an ideal model for translational research studies for various human diseases. Our review will specifically focus on endovascular ischemic stroke models in NHPs and discuss the use of NHPs in stroke research.

NHP stroke models are mainly produced by 2 methods: endovascular approaches and craniotomy. The open surgical models have advantages such as to lower variability and mortality because of precise blockage of the target vessel [13]. However, the traumatic aspect of invasive surgery might be viewed as a confounding factor because ischemic stroke in humans is not commonly associated with head trauma [14]. Compared with ischemic stroke models achieved by craniotomy in NHPs, ischemia induced by endovascular methods closely mimics thromboembolic or thrombotic cerebrovascular occlusion in patients. However, previous studies adopted various methods to induce focal ischemia in NHPs with variable outcomes. Owing to the shared features of pathophysiological mechanisms of ischemic stroke in NHPs and human patients, we will summarize NHPs models of focal cerebral ischemia developed by endovascular methods, and explore their respective strengths and weaknesses so as to describe the optimum model for translational studies.

\section{Ischemia Models in NHPs}

Based on previous studies and reports, an ideal ischemic stroke model in NHPs needs to meet 3 basic criteria: 1) formation of a relatively large infarct size; 2) notable neurologic impairments; and 3) a long-term survival period (at least 7 days) of the animal $[13,15,16]$. Since these models simulate the pathophysiological process of focal ischemia as seen in patients, they provide a unique opportunity for developing potential treatments.

Brains of gyrencephalic primates resemble human brains more closely than lissencephalic species. Thus, for the sake of simplicity, our review will primarily summarize studies using gyrencephalic NHPs, including commonly used Macaca fascicularis (cynomolgus monkey), Macaca mulatta (rhesus monkey) and Papio anubis (baboon) [5]

Several features of NHPs account for their great benefits in preclinical research. Genetically, Macaca and baboons share $92 \%$ of their genome sequence with that of humans, whereas mice and rats share only $66 \%$ and $64 \%$ of their genome with that of humans, respectively [10]. Anatomically, macaque monkeys and baboons have a gyrencephalic brain with similar cortical and subcortical structure to human brains. Moreover, NHPs also have a much higher percentage of white matter than mice, rat, and rabbits, making it similar to the white matter content in human brains [3, 17]. Further, NHPs have a complete cerebral arterial ring and distributions of the internal carotid and vertebral arteries as those in humans $[10,18]$.

Studies have shown that leukocyte composition, wholegenome mRNA, and microRNA expression in leukocytes, as well as inflammatory molecules expressed during stoke, differ in rodents compared with humans [19]. However, unlike rodents, NHPs have a similar immune composition, which responds to ischemic stroke as in humans [6]. Components of vascular hemostasis also share great similarities between NHPs and humans, including platelets, coagulation proteins, and fibrinolytic proteins [15]. Additionally, NHPs also have the ability to perform complex motor planning tasks just as in humans. Lastly, consequences of infarction of cerebral vessels are very similar to those in humans and are predictable, depending on the site of occlusion. For instance, infarction at the main trunk (M1) of the MCA in NHPs causes ischemia primarily in the basal ganglia and white matter, similar to patients with MCA stroke [15]. Moreover, ischemic damage of the white matter is a prognostic factor for stroke outcomes. However, only NHP stroke models are able to mimic white matter pathology in stroke patients most closely to pathology seen in humans and is recognized as an important limitation to rodent ischemic stroke models [20-24]. Thus, NHP stroke models have numerous intrinsic advantages over rodents, especially owing to their similarity in different fronts of the human anatomy and physiology, making them an irreplaceable and indispensable animal model for studying cerebral ischemia [25].

\section{Ethical Considerations}

While NHPs serve as the closest animal models to humans, the ethical challenges inherent in primate research must be carefully considered when designing a primate stroke model. To begin with, endovascular methods to induce ischemic stroke in NHPs, as a minimally invasive surgery, can eliminate or reduce traumatic pain when invasive surgery or craniotomy are adopted. However, it does not reduce pain, distress, or emotional anxiety due to neurological deficits following the onset of ischemia. Analgesia, environmental enrichment, treatment for antiedema and antibacterial infection, parenteral nutrition, and intensive veterinary care are basic requirements for NHP models [16, 26]. Another critical issue is to calculate the minimal number of animals needed to detect any significant effect of a neuroprotective treatment in NHP models [27]. 
This can be resolved by the research group by consulting a biostatistician, as well as the Institutional Animal Care and Use Committee. In addition, the Institutional Animal Care and Use Committee should also abide by the recommendations of the Stroke Therapy Academic Industry Roundtable, which states that stroke recovery studies in NHPs may be done with a gyrencephalic species, similar to humans (e.g., macaque monkeys) once sufficient evidence of efficacy has been obtained for a given therapy in rodent models $[5,28,29]$.

\section{Permanent MCA Occlusion}

Permanent models of stroke often involve blocking $\geq 1$ of the major cerebral arteries or simply leaving an intraluminal embolus in the artery (surgical suture or thrombus) [30]. In NHPs, permanent MCA occlusion is generally developed with emboli that could not be resolved with t-PA treatment, such as cyanoacrylate adhesive, nylon thread, silk sutures, and polystyrene spheres. The experimental paradigms using different materials for permanent occlusion models are summarized in Table 1 [26, 31-35].

Early studies used cyanoacrylate adhesive as emboli, which was injected to the distal branch of the MCA. These studies reported a large variation in infarct size $(3.38-20.5 \mathrm{ml})$, suggesting a possibility of migration of the final occlusion site from the original site of injection [26]. Thus, in order to restrict the occlusion site, later studies obstructed the distal M2 section of MCA using silk sutures as the emboli. This approach for permanent MCA occlusion successfully induced a reproducible and appropriate cortical infarction in adult rhesus monkeys [31]. In another study, multiple segments of silk sutures were first injected within the desired M3 branch, followed by a final suture injected into an M1 segment to control for collateral arteries. Such an approach, called endovascular trapping, successfully occluded collateral vessels to the desired vessel of occlusion (M3), leading to production of reproducible ischemia in MCA territory with clinically quantifiable neurologic deficits [32]. Such studies demonstrated the feasibility of producing a precise permanent occlusive model in NHPs with advancements in techniques and materials. Thus, radiographic data from the aforementioned study and those from stroke patients strongly suggest that precise control of occlusion site is an important prerequisite for a successful permanent MCA occlusion model in NHPs $[16,36]$.

Common in practice, most researchers chose to occlude the distal M1 or M2 for permanent models in order to preserve the perforating branches of lenticulostriate arteries [26, 31]. Moreover, studies have found that when the M1 trunk is blocked with glue or silk sutures, these models mimic largevessel ischemic stroke with an unfavorable prognosis. However, when a distal M1 or M2 segment is blocked, those models generally have a modest infarct size and do not cause a fatal ischemic stroke in the animal $[26,31]$. These discussed permanent models satisfy the 3 criteria for an ideal ischemic stroke model and have been successfully used to generate preclinical and basic science data for investigating promising neuroprotective therapies, novel restorative strategies, and brain plasticity in poststroke recovery [37].

An alternative approach for developing permanent occlusive models has been the injection of embolic spheres. In a study by Cook et al. [34], the authors injected polystyrene spheres into the ICA of cynomolgus monkeys to induce focal ischemia. They found that emboli with larger diameter spheres $(400 \mu \mathrm{m})$ produced severe neurological impairments due to ischemia in the basal ganglia and internal capsule, whereas spheres with a modest diameter $(200 \mu \mathrm{m})$ only induced a mild and lasting neurologic deficit; spheres with a smaller diameter $(100 \mu \mathrm{m})$ did not induce any noticeable neurologic deficit, even when used in large quantities [34]. In another study, a lacunar-type stroke model was developed in macaque monkeys by injecting agarose spheres $(50 \mu \mathrm{m}$ in diameter) into the ICA of these monkeys [35]. The discussed sphere-induced models of occlusion did not exhibit obvious neurological

Table 1 Summary of permanent middle cerebral artery occlusion (MCAo) in nonhuman primates

\begin{tabular}{|c|c|c|c|c|c|c|}
\hline Source & Species & Location & MCAo duration & Emboli & Size $(\mathrm{ml})$ & Outcome \\
\hline D'Arceuil et al. [26] & Cynomolgus & M1 & Permanent & Glue & $3.38-20.5$ & $\begin{array}{l}\text { Death }(\mathrm{n}=2) \\
\text { Survival }(\mathrm{n}=2)\end{array}$ \\
\hline Zhang et al. [31] & Rhesus & Distal M2 & Permanent & Silk suture & $1.08-7.8$ & $\begin{array}{l}\text { Death }(n=2) \\
\text { Sacrifice }(n=3)\end{array}$ \\
\hline Tong et al. [32] & Rhesus & M3 and M1 & Permanent & Silk suture & $3.0-12.9$ & $\begin{array}{l}\text { Death }(n=2) \\
\text { Sacrifice }(n=6)\end{array}$ \\
\hline Sato et al. [35] & Cynomolgus & ICA & $\begin{array}{l}\text { Permanent } \\
\quad \text { (lacunar stroke) }\end{array}$ & Sephacryl beads & NA & NA \\
\hline Cook et al. [34] & Cynomolgus & ICA & Permanent & Polystyrene spheres & $10-40$ & Death $(\mathrm{n}=1)$ Sacrifice $(\mathrm{n}=18)$ \\
\hline Rodriguez-Mercado et al. [33] & Rhesus & M1 & Permanent & Silk sutures & $0.2-15.0$ & Sacrifice $(n=3)$ \\
\hline
\end{tabular}

ICA = internal carotid artery; NA = not applicable 
impairments. Although the number and volume of infarct site could be determined based on magnetic resonance images, it is not possible to control for the occlusion site and retrieve spheres in these models. However, these models can be used to mimic small embolic strokes that occur in multiple clinical scenarios, such as cardiopulmonary bypass, carotid endarterectomy or stenting, and other endovascular procedures [34].

While permanent MCA occlusion models in NHPs can mimic pathological processes and outcomes in ischemic stroke, they precluded the possibility of t-PA thrombolysis and could not simulate the treatment process in ischemic patients. Thus, to understand the outcomes of treatments in ischemic stroke, transient MCA occlusion models are needed.

\section{Transient MCA Occlusion}

Transient MCA occlusion in NHPs is generally induced with a retrievable tool such as a microcatheter, an inflatable balloon, and a microcoil. Ischemia occurs as a result of consequent blockade of blood flow, whereas reperfusion is achieved when the tool is withdrawn. Transient MCA occlusion in NHPs by endovascular methods from various studies are summarized in Table 2 [14, 16, 26, 37-45].

It is relatively easy and cost friendly to create focal ischemia in NHPs with a microcatheter. A previous study reported a small infarct size in cynomolgus monkeys using a Prowler10 microcatheter $(0.55 \mathrm{~mm}$ outer diameter $)$ to block the distal branch of M1 [37]. The same method was also widely adopted to develop a reversible MCA occlusion model in NHPs [46]. However, the infarct size in this model was relatively small
(0.4-3.2 ml, Diffusion Weighted Imaging (DWI) at $3 \mathrm{~h}$ ) and there was no information about neurologic deficit after the onset of ischemia. To improvise upon this model, another study used a larger microcatheter (Rebar 18 microcatheter) to induce focal ischemia in NHPs. However, infarct sizes were significantly larger than the expected vascular territory and correlated with a significant neurologic deficit when the microcatheter was navigated into the M1 segment of the MCA in rhesus monkeys [16]. Such findings can be explained by the fact that a large microcatheter serves as both an embolus and irritant for the MCA. While it is difficult to induce a homogenous infarct size just by inserting a microcatheter, it is possible to control ischemia and reperfusion as needed, making this method desirable. Thus, in order to resolve the issue of heterogeneous infarct size with this model, a combination of a microcatheter and additional emboli can help control the occlusion site and duration.

Using a microcatheter for transient occlusion confers the advantage of delivering agents to the occlusion site. Thus, this approach makes it easy to evaluate the efficacy and feasibility of local or regional infusion of fibrinolytics, neuroprotective drugs, or ice-cold saline before or after the reperfusion [47]. In fact, studies have suggested that microcatheter delivery may provide more benefits for the analysis of penumbral freezing, prevention of reperfusion injury, and enhanced recanalization efficacy in NHPs models [47].

Ischemia and reperfusion in NHPs can also be induced by inflation and deflation of a catheter-delivered balloon into a blood vessel. In 2006, Gao et al. [42] developed a novel balloon catheter $(0.61 \mathrm{~mm}$ outer diameter for microcatheter, $1.1 \mathrm{~mm}$ outer diameter for a deflated balloon) to temporarily occlude and reperfuse the MCA by inflation and deflation of

Table 2 Summary of transient middle cerebral artery occlusion (MCAo) in nonhuman primates

\begin{tabular}{|c|c|c|c|c|c|c|}
\hline Source & Species & Location & $\begin{array}{l}\text { MCAo } \\
\text { duration }\end{array}$ & Approach & Size & Outcome \\
\hline Del Zoppo et al. [44] & Baboons & M1 & $3 \mathrm{~h}$ & Balloon & $3.2 \pm 1.5 \mathrm{~mL}$ & Survival $(\mathrm{n}=5)$ \\
\hline Hamberg et al. [43] & Baboons & M1 & $3 \mathrm{~h}$ & $\begin{array}{l}\text { Balloon }(n=4) \\
\text { Microcoil }(n=1)\end{array}$ & $475-880 \mathrm{~mm}^{2}$ & Sacrifice $(n=5)$ \\
\hline Guo et al. [45] & Rhesus & M1 & $2 \mathrm{~h}$ & Microcoil & NA & $\begin{array}{l}\text { Survival } \\
\qquad(\mathrm{n}=6)\end{array}$ \\
\hline de Crespigny et al. [37] & Cynomolgus & M2 & $3 \mathrm{~h}$ & Microcatheter & $0.3-1.9 \mathrm{ml}$ & Sacrifice $(n=4)$ \\
\hline Gao et al. [42] & Rhesus & M1 & $2 \mathrm{~h}$ & Balloon & NA & NA \\
\hline \multirow[t]{2}{*}{ Zhao et al. [14] } & \multirow[t]{2}{*}{ Rhesus } & M1 & $2 \mathrm{~h}$ & Microcoil & $9.8-24.5 \mathrm{ml}$ & NA \\
\hline & & M2 & $2 \mathrm{~h}$ & Microcoil & $1.6-3.1 \mathrm{ml}$ & NA \\
\hline Wu et al. [16] & Rhesus & M1 & $3 \mathrm{~h}$ & Microcatheter & $9.7-20.1 \mathrm{ml}$ & $\begin{array}{l}\text { Death }(\mathrm{n}=5) \\
\text { Survival }(\mathrm{n}=1)\end{array}$ \\
\hline Zhang et al. [38] & rhesus & M1 & $2 \mathrm{~h}$ & Microcoil & $2.95 \pm 0.48 \%$ & Survival $(\mathrm{n}=17)$ \\
\hline Yi et al. [39] & Rhesus & M2 & NA & Microcatheter & NA & Survival $(\mathrm{n}=7)$ \\
\hline Jungreis et al. [40] & Nemestrina & M1 & NA & Balloon & NA & NA \\
\hline Schwartz et al. [41] & Baboons & ACA & $3 \mathrm{~h}$ & Microcoil & $1.23,1.65 \mathrm{ml}$ & Survival $(\mathrm{n}=2)$ \\
\hline
\end{tabular}

$\mathrm{NA}=$ not applicable; $\mathrm{ACA}=$ anterior cerebral artery 
the balloon, respectively, in cynomolgus monkeys. The animals in this study exhibited hemiplegia and had a relatively large infarct size (mainly at basal ganglia and cortex) [42] Along similar lines, another study developed an ischemiaperfusion model to induce a 3 -h ischemic stroke using the catheter-based balloon technology discussed above. This group also reported relatively large stroke lesion sizes compared with other ischemic-reperfusion models in NHPs, demonstrating the robustness and reliability of this partial occlusion model [43]. Most importantly, transient ischemic stroke models form large lesion, causing neurological impairment along with a long survival period of the animal, satisfying the 3 key elements required to be an ideal stroke model, as discussed above. Although this model allows us to control the duration of occlusion, one of the main limitations of this model is that it only allows occlusion of the proximal MCA. This is due to the large diameter of the balloon catheter compared with that of the distal segments of MCA.

Another microcatheter-based MCA occlusion can be done via the introduction of a microcoil. A microcoil can be precisely positioned by advancing the microcatheter, and it can be repositioned until appropriate complete MCA occlusion is achieved. By introducing the microcoil into the M1, researchers can block blood flow into the blood vessel and can remove the coil to simulate reperfusion of M1. Studies have found that microcoil-induced M1 occlusion caused a large infarct in deep brain structures such as the basal ganglia and internal capsule, as well as the white matter and cortex [38, 45]. In addition, these models caused severe neurological impairments as seen as in stroke patients. In contrast to the neurological and anatomical outcomes with microcoil-induced M1 occlusion, M2 occlusion using the same technique actually caused a smaller infarct size, primarily in the cortex and the Sylvian fissure, and much milder neurological impairment [14]. The microcatheter-derived microcoil deployment method simulated the ischemia-reperfusion process in patients, in which the occlusion site and duration could be precisely controlled. In addition, this model also exhibited increased stability, safety, and reproducibility across various studies. Moreover, this transient ischemic stroke model qualifies as an ideal ischemic stroke model, as it satisfies the 3 required criteria as discussed above.

In all the transient ischemic stroke models discussed above, complete reperfusion is achieved when the retrievable tool is withdrawn in NHPs. However, in an intriguing study, when a balloon was used to induce focal ischemia in baboons, in situ thrombosis in perforating branches was observed [44]. Based on previous reports and our own experience, thrombosis and vasospasm could significantly affect ischemia size and clinical outcomes and thus it is important to keep these factors in mind when developing such models in NHPs [14]. All in all, transient MCA occlusion in NHPs is ideal for studying the effect of promising therapies that require proper reperfusion of the occluded cerebral vessel.

\section{Thrombus MCA Occlusion in NHPs}

Thrombi and emboli are the main causes for ischemic stroke in humans, with about $50 \%$ due to large-vessel atherosclerosis and $20 \%$ caused by cardioembolism [3]. Clot models in NHPs closely mimic the pathogenesis of stroke patients and hold potential for thrombolysis treatment. Thrombus models by endovascular methods are summarized in Table $3[16,40-58]$. In order to achieve an ideal ischemic stroke model in NHPs using thrombi, 2 key factors are needed: 1) accurate control of thrombus; and 2) timely recanalization of the vessel.

As reported in rodent models of thromboembolic stroke, the major concern of these models in NHPs is control over the introduced embolus, which may be determined by the site of occlusion and nature of the clot [55]. Such concerns arose when 2 studies reported an experimental thromboembolic model in NHPs in which the clots introduced were not at the injection site but migrated down to a nearby site [48, 49]. In order to selectively block the MCA, 1 study combined neurosurgical and endovascular methods in rhesus monkeys [50]. However, when the clot was delivered under pressure through a microcatheter, the clot was prone to break down owing to its fragile quality, compromising the model $[50,52]$. In a recent study, a single fibrin-rich clot was used to induce ischemia in rhesus monkeys, a technique derived from rodent models [16]. Using a single fibrin-rich clot significantly increased the toughness and flexibility of the clot as red blood cells and platelets were washed out during clot preparation [56]. As a result, clots did not break down when they were delivered from the microcatheter to the site of interest. Moreover, there was much better control over these clots with the aid of a microcatheter and were mainly located at M1 or M2 segment [16]. While it is challenging to control exactly the occlusion site using only the endovascular method of delivery, improved thrombus composition, a definite load (clot size), delicate operative skills, and a microcatheter-aided delivery increase the control of clot embolus significantly.

In spite of the omnipresence of ischemic stroke, thrombolysis with t-PA is the only approved treatment for patients with ischemic stroke, with a narrow therapeutic window of 3 to $4.5 \mathrm{~h}$ following onset of symptoms [47]. Successful recanalization with t-PA is imperative in both patients and NHPs when the MCA is permanently occluded by a clot $[16,57]$. However, only 1 paper reported that intra-arterial reteplase appeared to be effective in achieving recanalization in NHP models of intracranial thrombosis [54]. Unfortunately, small sample size and significant variability within each treatment group hindered 
Table 3 Summary of thrombus middle cerebral artery occlusion (MCAo) in nonhuman primates

\begin{tabular}{|c|c|c|c|c|c|c|}
\hline Source & Species & Location & Thrombolysis & Emboli & Size & Outcome \\
\hline Kito et al. [52] & Cynomolgus & M1 & No & Clot & $28.3 \pm 12.4 \%$ & Sacrifice $(\mathrm{n}=10)$ \\
\hline Qureshi et al. [54] & Rhesus & M1 & Yes, 2h (t-PA) & Thrombin injection & NA & Sacrifice $(\mathrm{n}=16)$ \\
\hline \multirow[t]{3}{*}{ Susumu et al. [53] } & \multirow[t]{3}{*}{ Cynomolgus } & M1 & No & Clot & $24.7 \pm 3.5 \%$ & Sacrifice $(\mathrm{n}=10)$ \\
\hline & & M1 & Yes, 2 h (UA) & Clot & $11.9 \pm 3.9 \%$ & Sacrifice $(n=6)$ \\
\hline & & M1 & Yes, 6 h (UA) & Clot & $7.6 \pm 2.5 \%$ & $\begin{array}{l}\text { Death }(n=2) \\
\text { Sacrifice }(n=3)\end{array}$ \\
\hline \multirow[t]{2}{*}{ Wu et al. [16] } & \multirow[t]{2}{*}{ Rhesus } & M1 & No & Clot & $7.7-13.5 \mathrm{ml}$ & $\begin{array}{l}\text { Death }(n=2) \\
\text { Survival }(n=2)\end{array}$ \\
\hline & & M2 & No & Clot & $3.2-7.1 \mathrm{ml}$ & Survival $(n=6)$ \\
\hline Kuge et al. [51] & Cynomolgus & ICA & No & Clot & NA & NA \\
\hline Xie et al. [49] & NA & ICA & No & Clot & NA & NA \\
\hline
\end{tabular}

$\mathrm{t}-\mathrm{PA}=$ tissue plasminogen activator; $\mathrm{NA}=$ not applicable; $\mathrm{UA}=$ urokinase; $\mathrm{ICA}=$ internal carotid artery

statistical analysis in this study. In another study, urokinase was reported to improve neurologic deficits and reduce cerebral infarction in the thromboembolic stroke model of cynomolgus monkey [53]. However, a high dose led to cerebral edema and hemorrhage despite a better neuroprotective effect. Moreover, another paper showed that urokinase reduced infarct volume owing to its thrombolytic effect on in situ thrombosis of perforating branches formed in a reversible eccentric balloon compression $(3 \mathrm{~h})$ baboon model [44]. While there are some promising studies favoring the use thrombolytics in NHPs, additional studies are needed to establish an algorithm, similar to the thrombolysis in myocardial infarction algorithm, to evaluate response to the thrombolysis treatment in NHPs and humans. Moreover, future studies are also needed that investigate safe administration of recombinant t-PA and therapeutic effect of recombinant t-PA following acute ischemia in NHPs.

In the past decade, 5 randomized trials showed efficacy of endovascular thrombectomy in patients with acute ischemic stroke caused by occlusion of arteries of the proximal anterior circulation [58]. Thrombus occlusion models in the proximal of MCA or ICA have been established in NHPs but have proven to be fatal without successful recanalization of the blood vessel $[16,52]$. Moreover, to date, there are no established NHPs models that simulate endovascular thrombectomy in humans. Thus, research to develop these models is imperative.

The greatest advantage of thromboembolic models is 2fold: 1) simulation of the pathogenesis of cerebrovascular occlusion and focal ischemia; and 2) the opportunity for recanalization treatment of occluded vessels. On the flip side, these models may not replicate the characteristics of spontaneous thrombus or thrombus superimposed on ruptured atherosclerotic plaque, conferring the data from such models limited.

\section{Infarct Size, Symptoms, and Prognosis}

Endovascular techniques appear to have increased variability in stroke size with a higher mortality rate than open vascular occlusions in focal ischemia models of NHPs [5]. Thus, many early studies only reported the infarct size and neurological examination scores in the acute state [5]. However, recent reports have found an association between infarct size and outcomes in NHP stroke models developed using endovascular methods [16, 26, 30,31]. Studies have previously reported the correlation between functional outcome and infarct size in both rodents and NHPs $[13,59,60]$. As a result, neurological impairments and prognosis in NHPs models are first characterized based on the infarct size, and then further analyzed for the application of behavioral tests in this paper. Studies have shown that when infarct size is $<3.0 \mathrm{ml}$, NHPs exhibit a mild, short-term functional deficit or abnormal behavior following recovery from anesthesia, according to Spetzler Scores, in transient and permanent MCA occlusion models $[26,34,37]$. These animals mimic patients with minor strokes showing abnormal radiographs but with or without functional deficit. On the contrary, when infarct size is $>15$ $\mathrm{ml}$, NHPs exhibit severe neurological impairments in transient, permanent, and thrombus MCA occlusion models, including symptoms of hemiplegia of contralateral limbs, coma or delirium, decreased muscle tone, and salivation [14, 16, 26]. These NHPs simulate clinical features of patients with severe stroke with a poor prognosis. Moreover, when infarct size is between 3.0 and $15.0 \mathrm{ml}$, the most common symptom observed is contralateral motor weakness in all transient, permanent, and thrombus MCA occlusion models [14, 32, 37, 44]. These animals mimic a majority of stroke survivors having a permanent functional impairment.

Stroke patients generally exhibit sensorimotor impairments and paralysis. In clinical studies of acute ischemic 
stroke, the modified Rankin Scale is used to assess functional status at 90 days following the stroke event or the index event [59]. The ability to perform motor planning tasks is unique to NHPs and humans, making NHP models extremely important to understand the functional implications of stroke. Behavioral tests for postischemic strokeinduced functional impairments have been reported in NHPs models undergoing craniotomy. These include a sensorimotor battery of tasks and object retrieval detour task $[13,61]$. Similar function tests could also be performed in NHP stroke models using endovascular methods when they meet all 3 criteria for an ideal NHP model. Thus, to reiterate, NHPs offer unique opportunities to model motor impairments in stroke patients and to understand cortical control of motor tasks in such patients.

\section{Abnormal Hemodynamics, Occlusion Site and Clinical Outcomes}

Platelets play an important role in the pathogenesis of cerebral ischemia. This begins with aggregation of platelets following their activation. Moreover, platelet secretory products have been implicated in the evolution of stroke [62]. As shown in Fig. 1, when the M2 segment of the MCA is permanently blocked with silk sutures (Fig. 1A)
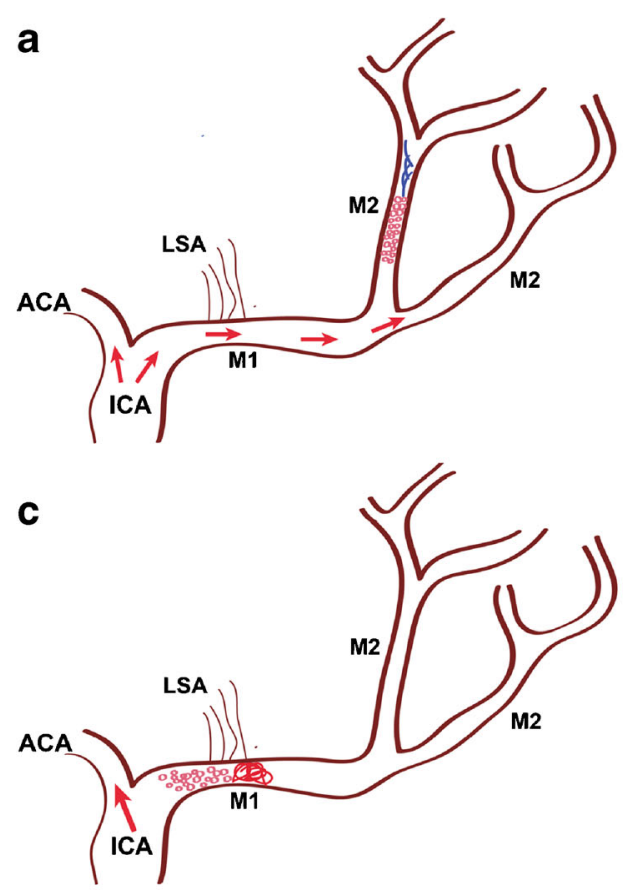

Fig. 1 The evolution of abnormal hemodynamics in different inducing methods. (A) When silk sutures (in blue) are injected to distal branch of M1 (M2), platelets (in pink) will aggregate to the proximal of M2. (B) When a clot (in red) is injected to M2, platelets (in pink) will aggregate to the proximal of M2. Normal blood flow is preserved. (C) When a clot (in red) is injected to main trunk of middle cerebral artery (M1), platelets (in or a clot (Fig. 1B), there is normal blood flow from the ICA to M1, and subsequent blood flow to the second branch of M2. Thus, a local perfusion defect will lead to focal ischemia and mild neurological deficit. However, when M1 segment is blocked with a clot (Fig. 1C) or a retrievable tool (Fig. 1D), there is normal blood flow only from the ICA to the anterior cerebral artery and not to MCA branches, as expected. When reperfusion is achieved in these models using thrombolysis treatment or by the withdrawal of the tool, infarcts are primarily found at the putamen and internal capsule, supplied by the horizontal segment of MCA [48]. Fortunately, these 4 models discussed above meet all 3 criteria for an ideal NHP model, validating their use in research. However, researchers should keep in mind that unexpected abnormal hemodynamics may occur in rare cases. Figure 2 shows abnormal hemodynamics in an NHP model, caused by injecting a clot. Immediately after injection of a clot into the MCA, a perfusion deficit was initially observed in the proximal segment of the artery. However, $3 \mathrm{~h}$ later this clot extended into the ICA, blocking the perfusion of regions beyond the occluded ICA. As a result of the abnormal blockade of ICA, these NHPs will exhibit severe neurological impairments.

Pathologically, these NHPs models exhibited cerebral edema, which was significant within 7 days following the onset of ischemia both in stroke patients and NHP models
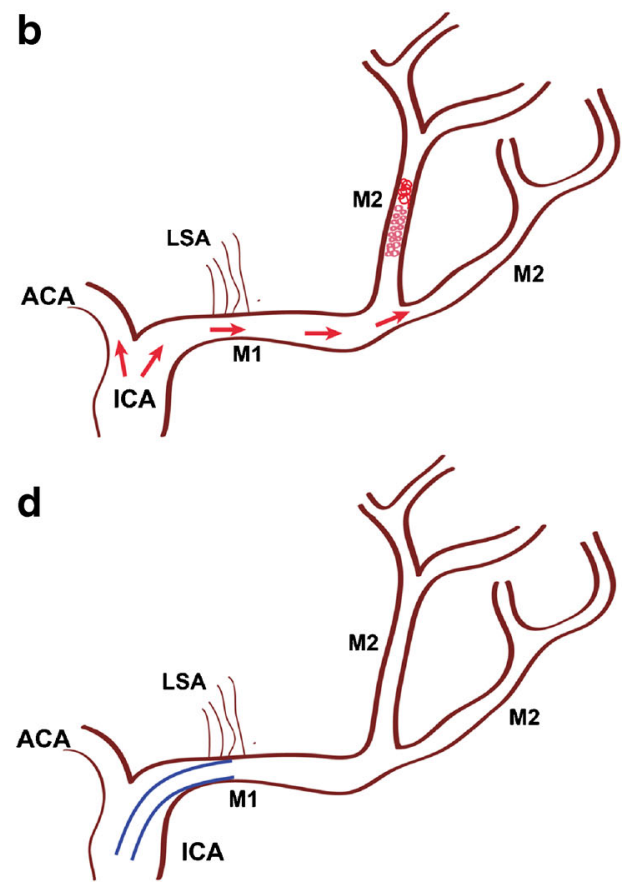

pink) will aggregate to the proximal of M1. Blood flow from MCA will only go to the anterior cerebral artery (ACA). (D) when a retrievable tool (e.g., a microcatheter in blue) is inserted at M1 segment of the middle cerebral artery (MCA), blood flow from the MCA will only go to the ACA. LSA = lenticulostriate arteries 
Fig. 2 The thrombus extended to internal carotid artery (ICA) following the original injection of a clot at main trunk of middle cerebral artery (M1). (A) Anteriorposterior and (B) lateral views of normal perfusion in adult rhesus monkey when the microcatheter was placed at the main trunk (M1) of middle cerebral artery (MCA). (C) Anterior-posterior and (D) lateral views showing perfusion deficit in the proximal end of MCA just following the injection of clot. (E) Anterior-posterior and (F) lateral views exhibiting no perfusion in the ICA segment at $3 \mathrm{~h}$ following the injection of clot
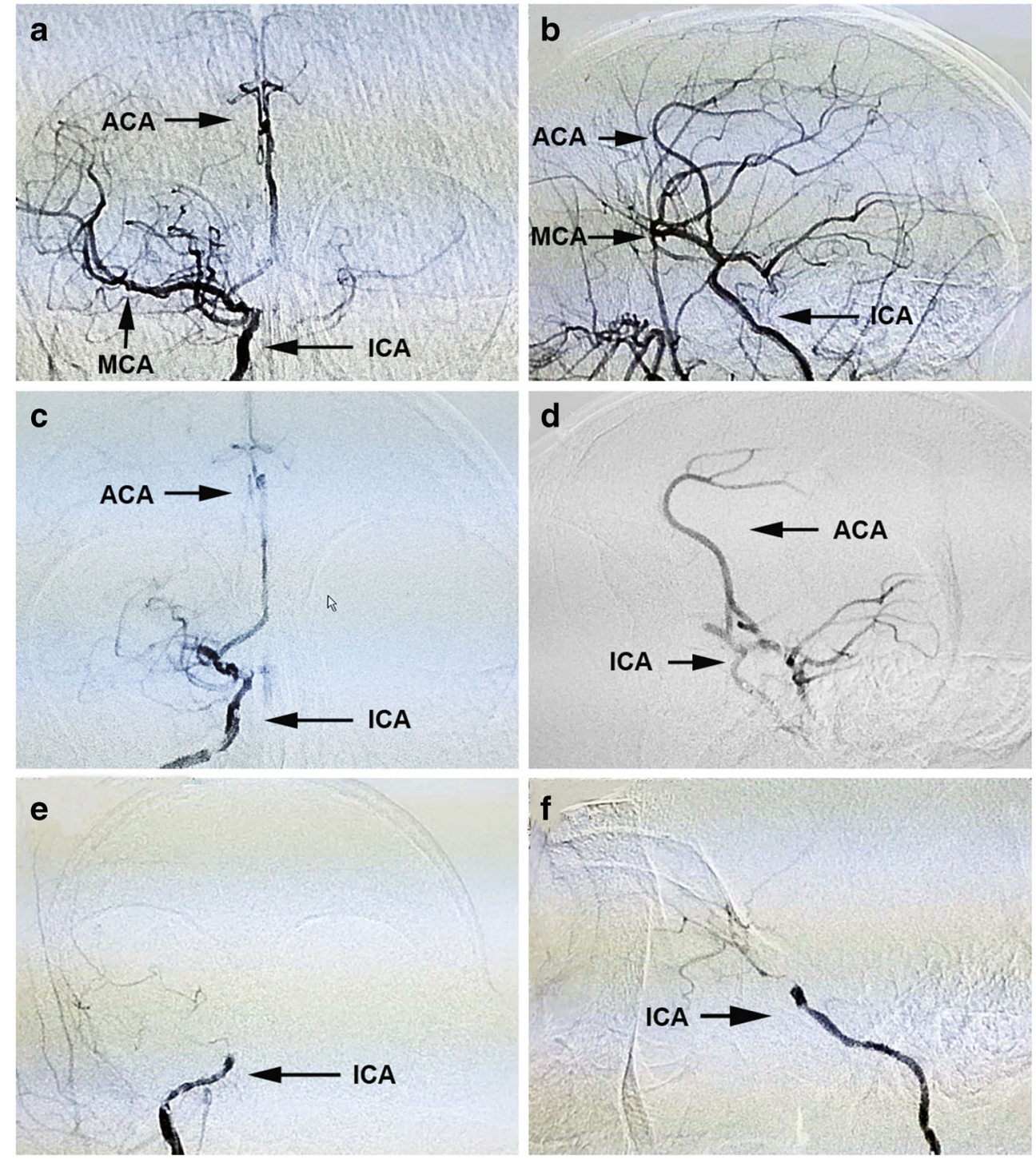

$[26,63]$. Clinically, this time period correlated with a higher mortality rate in both humans and NHPs, giving us a deeper understanding of the implications of poststroke edema $[26,57]$.

Lastly, it is important to note that although there are many reports on NHP models of stroke focusing in anterior cerebral circulation, those models almost exclusively focus on MCA occlusion. Only few studies reported vertebral artery or basilar artery occlusion models in NHPs with endovascular techniques. Based on reports from canine models, studies have found that vertebral or basilar artery occlusion models exhibited a severe and fatal neurological deficit, making it difficult to study the clinical and pathophysiological effects of such strokes [64]. Thus, there is a need to develop additional NHP models that can help simulate clinical consequences of vertebral and basilar artery strokes in human. International cooperation in preclinical stroke research, especially in NHPs models, is needed [65].

\section{Conclusions}

Ischemic stroke is a complex and heterogeneous disorder that affects a large population in the world. Ischemic stroke is also a long-term pathologic process, and includes 3 phases: 1) acute, 2) subacute; and 3) chronic. Experimental models of ischemic stroke are valuable tools with which to analyze specific clinical, pathological, and morphological facets of stroke paralleling those in humans. Over the years, several NHPs stroke models have been developed using various different techniques. Each NHPs model has its own pros and cons, and thus we should select the appropriate NHPs model based on the goals of our study and outcomes we would like to measure and analyze in a preclinical context. Among other animal models, NHPs models provide the most valuable predictions for human trials due to the anatomical, immunological, and pathophysiological homology between NHPs and humans. As the mainstay of NHP scientific research and 
potential preclinical studies, M. fascicularis (cynomolgus monkey) and M. mulatta (rhesus monkey), and the clot method are recommended as preferred species and the appropriate method for testing human neuroprotectants in translational stroke studies, respectively [50, 66].

Acknowledgments This work was supported by National Natural Science Foundation of China for Outstanding Youth (81325007); National Natural Science Foundation of China (81500997, 81771260); Chang Jiang Scholars Program (\#T2014251) from the Chinese Ministry of Education; National Natural Science Foundation of China (81620108011); National Key R\&D Program of China (2017YFC1308401); and the "mission" talent project of Beijing Municipal Administration of Hospitals (SML20150802); Beijing Municipal Administration of Hospitals' Youth Programme (QML20170802). Ankush Chandra is a Howard Hughes Medical Institute Medical Research Fellow.

\section{References}

1. Truelsen T, Begg S, Mathers C. The global burden of cerebrovascular disease. Geneva: World Health Organization, 2000.

2. Mozaffarian D, Benjamin EJ, Go AS, et al. Executive summary: heart disease and stroke statistics - 2016 update: a report from the American Heart Association. Circulation 2016;133:447-454.

3. Sommer CJ. Ischemic stroke: experimental models and reality. Acta Neuropathol 2017;133:245-261.

4. O'Collins VE, Macleod MR, Donnan GA, Horky LL, van derWorp $\mathrm{BH}$, Howells DW. 1,026 experimental treatments in acute stroke. Ann Neurol 2006;59:467-477.

5. Cook DJ, Tymianski M. Nonhuman primate models of stroke for translational neuroprotection research. Neurotherapeutics 2012;9: 371-379.

6. Herson PS, Traystman RJ. Animal models of stroke: translational potential at present and in 2050 Future Neurol 2014;9:541-551.

7. Jickling GC, Sharp FR. Improving the translation of animal ischemic stroke studies to humans Metab Brain Dis 2015;30:461-467.

8. Wu D, Yue F, Zou C, Chan P, Zhang YA. Analysis of glucose metabolism in cynomolgus monkeys during aging. Biogerontology 2012;13:147-155.

9. Kemnitz JW. Calorie restriction and aging in nonhuman primates. ILAR J 2011;52:66-77.

10. Grow DA, McCarrey JR, Navara CS. Advantages of nonhuman primates as preclinical models for evaluating stem cell-based therapies for Parkinson's disease. Stem Cell Res 2016;17:352-366.

11. Bruns CM, Baum ST, Colman RJ, Kemnitz JW, Weindruch R, Abbott DH. Insulin resistance and impaired insulin secretion in prenatally androgenized male rhesus monkeys. J Clin Endocrinol Metab 2004;89:6218-6223.

12. Colman RJ, Anderson RM, Johnson SC, et al. Caloric restriction delays disease onset and mortality in rhesus monkeys. Science 2009; 325:201-204.

13. Cook DJ, Teves L, Tymianski M. Treatment of stroke with a PSD95 inhibitor in the gyrencephalic primate brain Nature 2012;483: 213-217.

14. Zhao B, Zhao B, Shang G, et al. A more consistent intraluminal rhesus monkey model of ischemic stroke. Neural Regen Res 2014;9:2087-2094.

15. Fukuda S, del Zoppo GJ. Models of focal cerebral ischemia in the nonhuman primate. ILAR J 2003;44:96-104.
16. Wu D, Chen J, Wang B, et al. Endovascular ischemic stroke models of adult rhesus monkeys: a comparison of two endovascular methods. Sci Rep 2016;6:31608.

17. Borlongan CV, Jolkkonen J, Detante O. The future of stem cell therapy for stroke rehabilitation. Future Neurol 2015;10: 313-319.

18. Chandra A, Li WA, Stone CR, Geng X, Ding Y. The cerebral circulation and cerebrovascular disease I: Anatomy. Brain Circ 2017;3:45-56.

19. Sharp FR, Jickling GC. Modeling immunity and inflammation in stroke: differences between rodents and humans? Stroke 2014;45: e179-e180.

20. Bihel E, Roussel S, Toutain J, Bernaudin M, Touzani O. Diffusion tensor MRI reveals chronic alterations in white matter despite the absence of a visible ischemic lesion on conventional MRI: a nonhuman primate study Stroke 2011;42:1412-1419.

21. Sozmen EG, Hinman JD, Carmichael ST. Models that matter: White matter stroke models. Neurotherapeutics 2012;9: 349-358.

22. Hara K, Yasuhara T, Matsukawa N, et al. Hippocampal CA1 cell loss in a non-human primate model of transient global ischemia: a pilot study. Brain Res Bull 2007;74:164-171.

23. Hara K, Yasuhara T, Maki M, et al. Anomaly in aortic arch alters pathological outcome of transient global ischemia in Rhesus macaques. Brain Res 2009;1286:185-191.

24. Shindo A, Liang AC, Maki T, et al. Subcortical ischemic vascular disease: Roles of oligodendrocyte function in experimental models of subcortical white-matter injury. J Cereb Blood Flow Metab 2016; 36: 187-198.

25. Fan J, Li Y, Fu X, Li L, Hao X, Li S. Nonhuman primate models of focal cerebral ischemia. Neural Regen Res 2017;12:321-328.

26. D'Arceuil HE, Duggan M, He J, Pryor J, de Crespigny A. Middle cerebral artery occlusion in Macaca fascicularis: acute and chronic stroke evolution. J Med Primatol 2006;35:78-86.

27. Muller PY, Brennan FR. Safety assessment and dose selection for first-in-human clinical trials with immunomodulatory monoclonal antibodies. Clin Pharmacol Ther 2009;85:247-258.

28. Stroke Therapy Academic Industry Roundtable (STAIR). Recommendations for standards regarding preclinical neuroprotective and restorative drug development. Stroke 1999;30:2752-2758.

29. Fisher M, Feuerstein G, Howells DW, et al. Update of the stroke therapy academic industry roundtable preclinical recommendations. Stroke 2009;40:2244-2250.

30. Krieger DW, Yenari MA. Therapeutic hypothermia for acute ischemic stroke: what do laboratory studies teach us? Stroke 2004;35: 1482-1489.

31. Zhang X, Tong F, Li CX, et al. Temporal evolution of ischemic lesions in nonhuman primates: a diffusion and perfusion MRI study PLOS ONE 2015;10:e0117290.

32. Tong FC, Zhang X, Kempf DJ, et al. An enhanced model of middle cerebral artery occlusion in nonhuman primates using an endovascular trapping. AJNR Am J Neuroradiol 2015;36:23542359.

33. Rodriguez-Mercado R, Ford GD, Xu Z, et al. Acute neuronal injury and blood genomic profiles in a nonhuman primate model for ischemic stroke Comp Med 2012;62:427-438.

34. Cook DJ, Teves L, Tymianski M. A translational paradigm for the preclinical evaluation of the stroke neuroprotectant Tat-NR2B9c in gyrencephalic nonhuman primates. Sci Transl Med 2012;4: 154 ra133.

35. Sato Y, Chin Y, Kato T, et al. White matter activated glial cells produce BDNF in a stroke model of monkeys Neurosci Res 2009; 65:71-78.

36. Wein TH, Bornstein NM. Stroke prevention: cardiac and carotidrelated stroke. Neurol Clin 2000;18:321-341.

37. de Crespigny AJ, D'Arceuil HE, Maynard KI, et al. Acute studies of a new primate model of reversible middle cerebral artery occlusion J Stroke Cerebrovasc Dis 2005; 14:80-87. 
38. Zhang Y, Fan F, Zeng G, et al. Temporal analysis of blood-brain barrier disruption and cerebrospinal fluid matrix metalloproteinases in rhesus monkeys subjected to transient ischemic stroke. J Cereb Blood Flow Metab 2017;37: 2963-2974.

39. Yi KS, Choi CH, Lee SR, et al. Sustained diffusion reversal with inbore reperfusion in monkey stroke models: Confirmed by prospective magnetic resonance imaging J Cereb Blood Flow Metab 2017:37:2002-2012.

40. Jungreis CA, Nemoto E, Boada F, Horowitz MB. Model of reversible cerebral ischemia in a monkey model. AJNR Am J Neuroradiol 2003;24:1834-1836.

41. Schwartz AE, Pile-Spellman J. New model of reperfused stroke by occlusion of the anterior cerebral artery in baboons Acta Neurochir (Wien) 2011;153:327-331.

42. Gao H, Liu Y, Lu S, Xiang B, Wang C. A reversible middle cerebral artery occlusion model using intraluminal balloon technique in monkeys. J Stroke Cerebrovasc Dis 2006;15:202-208.

43. Hamberg LM, Hunter GJ, Maynard KI, et al. Functional CT perfusion imaging in predicting the extent of cerebral infarction from a 3 hour middle cerebral arterial occlusion in a primate stroke model. AJNR Am J Neuroradiol 2002;23:1013-1021.

44. Del Zoppo GJ, Copeland BR, Harker LA, et al. Experimental acute thrombotic stroke in baboons. Stroke 1986;17:1254-1265.

45. Guo J, Zheng HB, Duan JC, et al. Diffusion tensor MRI for the assessment of cerebral ischemia/reperfusion injury in the penumbra of non-human primate stroke model. Neurol Res 2011;33:108-112.

46. LaVerde GC, Jungreis CA, Nemoto E, Boada FE. Sodium time course using $23 \mathrm{Na}$ MRI in reversible focal brain ischemia in the monkey J Magn Reson Imaging 2009;30:219-223.

47. Fisher M, Saver JL. Future directions of acute ischaemic stroke therapy Lancet Neurol 2015;14:758-767.

48. Molinari GF, Moseley JI, Laurent JP. Segmental middle cerebral artery occlusion in primates: an experimental method requiring minimal surgery and anesthesia. Stroke 1974;5:334-339.

49. Xie Y, Munekata K, Seo K, Hossmann KA. Effect of autologous clot embolism on regional protein biosynthesis of monkey brain Stroke 1988;19:750-757.

50. Gauberti M, Obiang P, Guedin P, et al. Thrombotic stroke in the anesthetized monkey (Macaca mulatta): characterization by MRI-a pilot study. Cerebrovasc Dis 2012;33:329-339.

51. Kuge Y, Yokota C, Tagaya M, et al. Serial changes in cerebral blood flow and flow-metabolism uncoupling in primates with acute thromboembolic stroke J Cereb Blood Flow Metab 2001;21:202-210.

52. Kito G, Nishimura A, Susumu T, et al. Experimental thromboembolic stroke in cynomolgus monkeys. J Neurosci Methods 2001;105:45-53.

53. Susumu T, Yoshikawa T, Akiyoshi Y, Nagata R, Fujiwara M, Kito G. Effects of intra-arterial urokinase on a non-human primate thromboembolic stroke model. J Pharmacol Sci 2006;100:278-284.
54. Qureshi AI, Suri MF, Ali Z, et al. Intraarterial reteplase and intravenous abciximab for treatment of acute ischemic stroke. A preliminary feasibility and safety study in a nonhuman primate model. Neuroradiology 2005;47:845-854.

55. Orset C, Macrez R, Young AR, et al. Mouse model of in situ thromboembolic stroke and reperfusion Stroke 2007;38:2771-2778.

56. Zhang L, Zhang RL, Jiang Q, Ding G, Chopp M, Zhang ZG. Focal embolic cerebral ischemia in the rat Nat Protoc 2015;10:539-547.

57. Hacke W, Schwab S, Horn M, Spranger M, De Georgia M, von Kummer R. 'Malignant' middle cerebral artery territory infarction: clinical course and prognostic signs. Arch Neurol 1996;53:309-315.

58. Goyal M, Menon BK, van Zwam WH, et al. Endovascular thrombectomy after large-vessel ischaemic stroke: a meta-analysis of individual patient data from five randomised trials. Lancet 2016;387:1723-1731.

59. Banks JL, Marotta CA. Outcomes validity and reliability of the modified Rankin scale: implications for stroke clinical trials: a literature review and synthesis. Stroke 2007;38:1091-1096.

60. Rogers DC, Campbell CA, Stretton JL, Mackay KB. Correlation between motor impairment and infarct volume after permanent and transient middle cerebral artery occlusion in the rat. Stroke 1997;28: 2060-2066.

61. McEntire CR, Choudhury GR, Torres A, Steinberg GK, Redmond DE Jr, Daadi MM. Impaired arm function and finger dexterity in a nonhuman primate model of stroke: motor and cognitive assessments Stroke 2016;47:1109-1116.

62. Joseph R, Welch KM, Oster SB, Grunfeld S, D'Andrea G A plasmatic factor may cause platelet activation in acute ischemic stroke. Circ Res 1989;65:1679-1687.

63. Neugebauer H, Witsch J, Zweckberger K, Jüttler E. Spaceoccupying cerebellar infarction: complications, treatment, and outcome Neurosurg Focus 2013;34:E8.

64. Atchaneeyasakul K, Guada L, Ramdas K, et al. Large animal canine endovascular ischemic stroke models: a review. Brain Res Bull 2016;127:134-140.

65. Dirnagl U, Hakim A, Macleod M, et al. A concerted appeal for international cooperation in preclinical stroke research. Stroke 2013;44:1754-1760.

66. Cyranoski D. Monkey kingdom. Nature 2016;532:300-302.

\section{Required Author Forms}

Disclosure forms provided by the authors are available with the online version of this article. 